\title{
Smoking Comorbidity in Alcoholism: Neurobiological and Neurocognitive Consequences
}

\author{
Dieter J. Meyerhoff, Yousef Tizabi, Julie K. Staley, Timothy C. Durazzo, Jennifer M. Glass, \\ and Sara Jo Nixon
}

\begin{abstract}
Considerable research attests to the adverse effects of chronic smoking on cardiac, pulmonary, and vascular function as well as on increased risk for various cancers. However, comparatively little is known about the effects of chronic smoking on brain function. Although smoking rates have decreased in the developed world (they have increased in the developing world), smoking rates have been at a persistently high level in individuals with alcohol use disorders. Despite the high prevalence of comorbid chronic smoking and alcohol dependence, very few studies have addressed the separate and interactive effects that smoking and alcoholic drinking may have on neurobiology and brain function. This symposium, which took place at the annual meeting of the Research Society on Alcoholism in Santa Barbara, California, on June 29, 2005, postulates that the neurobiologic and neurocognitive abnormalities commonly described in studies of alcohol-dependent individuals are modulated by concurrent abuse of tobacco products and that brain recovery in abstinent alcoholic individuals is affected by chronic smoking. Four expert speakers and a discussant from different research disciplines focus in this symposium on the description of neurobiological and neurobehavioral effects because of concomitant drinking and smoking. Understanding the potential separate effects and interactions of chronic nicotine/smoking and alcohol consumption promotes a better understanding of specific mechanisms and neurocognitive consequences of brain injury and brain recovery with abstinence. The material presented contributes useful information to ongoing discussions about treatment strategies for these comorbid disorders and valuable educational material that can be used to affect public perception about smoking and perhaps health policy.
\end{abstract}

Key Words: Nicotine, Cigarette Smoking, Comorbidity, Neurocognition, Neuroimaging.

$\mathrm{T}$ HERE WERE APPROXIMATELY 1.3 billion smokers worldwide in 2003 (World Bank 2003) and about 4.9 million premature deaths due to smoking-related illnesses in 2000 (World Health Organization, WHO). The majority of these occurred in the developed world (North America, Europe, Australia, East Asia, and former USSR), where death rates for tobacco use among 35- to 69-year-old males are $>25 \%$. The WHO called this sad phenomenon rather fittingly "Dying in Your Prime." We have not, however, reached the apex of this pandemic: in

From the Department of Radiology, University of California at San Francisco, San Francisco, California (DJM); the Department of Veteran's Affairs Medical Center, San Francisco, California (DJM, TCD); the Department of Pharmacology, Howard University, Washington, DC (YT); the Department of Psychiatry, Yale University, the New Haven, Connecticut (JKS); the Addiction Research Center, University of Michigan, Ann Arbor, Michigan (JMG); and the Department of Psychology, University of Kentucky, Lexington, Kentucky (SJN).

Received for publication September 30, 2005; accepted November 16, 2005.

Proceedings of a symposium presented at the annual meeting of the Research Society on Alcoholism, June 2005, Santa Barbara, CA.

Reprint requests: Dieter J. Meyerhoff, Dr. rer. nat., MR Unit, 4150 Clement Street 114M, San Francisco, CA 94121;Fax: 415-668-2864; E-mail:djmey@itsa.ucsf.edu.

Copyright (C) 2006 by the Research Society on Alcoholism.

DOI: $10.1111 /$ j.1530-0277.2006.00034.x
2020, there will be an estimated 10 million deaths/year from smoking (WHO 2002: World Health Report; Peto and Lopez, 1996), primarily because of dramatically increasing smoking rates in Asia. In the United States, approximately $23 \%$ of the adult population smoke (Center for Disease Control, CDC 2001: Report of the Surgeon General). 440,000 Americans die from cigarette smoking yearly (CDC: 2004: Report of the Surgeon General), which accounts for about $20 \%$ of all deaths and thereby constitutes the leading cause of adult deaths.

The developed world largely recognizes the devastating effects that chronic cigarette smoking has on pulmonary, cardiac, and vascular function and on the dramatically increased risks for various forms of cancer. This is in part because of aggressive campaigns by WHO and other widereaching health organizations to curb the use of tobacco products and to raise awareness about the negative consequences of chronic smoking on almost all organs and organ systems of the human body (see e.g., The Smoker's Body in COLORS Magazine 1997). Over the past decades, smoking rates within the general populations of nations across the developed world have dropped dramatically. However, smoking is on the rise in developing nations and smoking rates in the developed world remain very high among individuals with alcohol use disorder (AUD) and other neuropsychiatric disorders (e.g., schizophrenia, bipolar disorder). 
Approximately $50 \%$ to $80 \%$ of alcohol-dependent individuals smoke regularly, they demonstrate greater alcohol consumption than nonsmoking alcoholic individuals (Hurt et al., 1994; Pomerleau et al., 1997; Romberger and Grant, 2004), and $50 \%$ to $90 \%$ (depending on the study) of alcoholic individuals are nicotine-dependent (Room, 2004). Furthermore, most alcoholic individuals who smoke continue to demonstrate a high level of nicotine dependence during abstinence from alcohol (Currie et al., 2001). Chronic cigarette smoking in alcoholic individuals is associated with significantly higher quantity and frequency of alcohol consumption compared with nonsmoking or former-smoking alcohol-dependent individuals (York and Hirsch, 1995), and animal studies showed that nicotine increases voluntary alcohol intake (Lê et al., 2003). Alcoholic smokers have more severe nicotine dependence and greater difficulties quitting than nonalcoholic smokers (Romberger and Grant, 2004). In a US cohort treated for alcoholism, mortality associated with cigarette smoking was $51 \%$, whereas mortality related to alcohol-induced diseases alone was about 34\% (Hurt et al., 1996). More than 4 times as many Americans die from smoking-related than from alcohol-related causes (Miller and Gold 1998; Peto and Lopez, 1996).

Despite these staggering statistics and sobering knowledge about this tragic comorbidity, very little is known about the biologic and functional consequences of chronic smoking on brain function in AUD. Chronic, heavy alcohol consumption has been associated over the past 20 years with abnormalities in brain pathology and morphology, cerebral glucose and amino acid metabolism, receptor activity, molecular and cellular function, and neurocognition. However, to the best of our knowledge, none of these research studies have considered chronic smoking as a potential confound or as a factor that could possibly explain some of the variance associated with brain injury attributed primarily to alcoholism.

This symposium addressed the following questions: does chronic smoking contribute to detectable brain injury in alcoholic individuals? If so, in what way and how much? Does chronic smoking affect recovery from alcoholism? And if so, should this knowledge inform current neuropharmaceutical treatments aimed at modifying drinking behavior? After all, some of the same pharmacotherapies (e.g., naltrexone) that appear to control or modify outcomes of alcohol abuse may also reduce smoking levels in alcoholic smokers (Rohsenow et al., 2003). Thus, the focus of this symposium was on the description of the neurobiological and neurobehavioral effects of concomitant alcohol consumption and smoking. The presenters were united in their message that understanding the potential separate effects and interactions of chronic nicotine/smoking and alcohol consumption on the brain will not only help elucidate specific mechanisms of brain injury and recovery as well as their neurocognitive consequences, but will also contribute useful information to ongoing discussions about treatment strategies for these comorbid disorders.

Various theories try to explain the high comorbidity of smoking and alcoholic drinking. These include nicotine's and alcohol's mutually potentiating rewards, nicotine counteracting the adverse effects of alcohol on cognition and motor incoordination ("self-medication"), and a common genetic susceptibility, with smoking possibly sharing some of the same genetic determinants with alcoholism. Hepatic and brain cytochrome P450 2E1 metabolism of both alcohol and nicotine can generate reactive oxygen species, leading to oxidative damage to cellular structures and macromolecules including proteins, membrane lipids, carbohydrates, and DNA (Moriarty et al., 2003) and activation of tobacco-derived procarcinogens and nervous tissue pathologies (Howard et al., 2003a, 2003b). Dr. Yousef Tizabi from Howard University in Washington, DC, in the first presentation, addressed some of these issues by giving an overview of the basic interactions between alcohol and nicotine. Modulation of the inhibitory gamma amino butyric acid (GABA) system by alcohol is implicated in the development of alcohol tolerance, dependence, and withdrawal. Dr. Julie Staley from Yale University presented recent single photon emission computed tomography (SPECT) studies that suggest differential regulation of cortical benzodiazepine (BZ)$\mathrm{GABA}_{\mathrm{A}}$-receptors in alcoholic smokers versus nonsmokers in short-term sobriety. Receptor adaptations in alcohol dependence may contribute to the comorbidity between alcoholism and smoking and, more importantly, suggest that BZs commonly used to treat alcohol withdrawal symptoms may be differentially effective in alcoholic individuals who smoke versus those who do not. Dr. Timothy Durazzo from the DVA Medical Center San Francisco described magnetic resonance-derived morphometric and metabolic differences in the brains of smoking and nonsmoking alcohol-dependent individuals during early recovery from alcoholism and their relationships with select neuropsychological measures. Dr. Jennifer Glass from the University of Michigan provided a more detailed description of the neurocognitive effects of alcoholism and long-term smoking in a community-recruited sample of alcoholic men and controls. Dr. SaraJo Nixon from the University of Kentucky ended the symposium with some closing remarks that further supported the urgent need for more smoking-related research in alcoholism.

\section{AN OVERVIEW OF BASIC INTERACTIONS BETWEEN ALCOHOL AND NICOTINE}

\section{Yousef Tizabi, Vera C. Campbell, and Robert E. Taylor}

Despite numerous epidemiological indications of synergistic increases in risks of various cancers, e.g., those of the head, neck, and esophagus as well as duodenal ulcer, the incidence of concomitant smoking and drinking remains high. Theoretically at least, there could be 
3 factors or mechanisms contributing to this comorbidity. First, as alcohol and nicotine are strong reinforcers individually, combining the 2 may yield additive or synergistic rewarding effects (Tizabi et al., 2002). Second, pharmacological interactions between alcohol and nicotine (e.g., metabolism and receptor stimulation/inhibition) could play a significant role (Howard et al., 2003a, 2003b; Narahashi et al., 2001). Finally, there may be genetic factors contributing to concomitant addiction to alcohol and nicotine. Indeed, various studies are suggestive of the existence of common genes that can make an individual susceptible to becoming dependent on both alcohol and nicotine ( $\mathrm{Li}$ et al., 2005; Madden and Heath, 2002).

Support for possible additive rewarding effects of alcohol and nicotine is provided by studies demonstrating activation of mesolimbic dopamine pathway by each drug individually and in combination. Activation of the mesolimbic system, reflected in dopamine release from the nucleus accumbens, is believed to be intimately involved in reinforcing effects of drugs (see Pierce and Kumaresan, 2005 , for a recent review). We have observed the additive effects of alcohol and nicotine on dopamine release from the nucleus accumbens shell (NACC). Thus, submaximal doses of systemic alcohol, when combined with submaximal doses of nicotine injected directly into the ventral tegmental area (VTA), resulted in additive release of dopamine in NACC (Tizabi et al., 2002). This effect was also seen when combining systemic alcohol with systemic nicotine (Tizabi et al., 2004a). Moreover, the stimulatory effects of alcohol on dopamine release was blocked by injection or infusion of the nicotinic receptor antagonist mecamylamine into the VTA, suggesting that at least some of the reinforcing effects of alcohol may be mediated through its interactions with the nicotinic receptors in VTA (Ericson et al., 1998; Tizabi et al., 2002). This contention is further supported by studies demonstrating a suppressive effect of mecamylamine on alcohol intake in rats (Ericson et al., 1998; Larsson and Engel, 2004; Lê et al., 2000). Several human studies have also provided evidence for mecamylamine-induced attenuation of the euphoric or stimulant effects of alcohol as well as a decrease in the desire to consume more alcohol (Blomqvist et al., 2002; Young et al., 2005).

The reinforcing effects of a drug of abuse may be enhanced if the drug, in addition to a euphoric effect, also possesses an analgesic or antinociceptive effect. In this regard, both alcohol and nicotine have been shown to have analgesic properties. An additive or synergistic antinociceptive effect of alcohol and nicotine may further contribute to their coabuse. Recently, we have observed a supraadditive antinociceptive effect of alcohol and nicotine combination. This effect was manifested in both spinal- and supraspinal-mediated analgesia where ineffective doses of alcohol and nicotine, when combined, resulted in antinociception. Thus, in both tail flick (spinally mediated nociception) and hot plate (supraspinally mediated nociception) tests, acute administration of lower doses of alcohol and nicotine, which had no antinociceptive effects alone, when combined resulted in significant antinociception (Campbell et al., 2005). These preliminary findings indicate that the combined reinforcing effects of alcohol and nicotine may be a contributory factor to their coabuse.

It is now well documented that neuronal nicotinic receptors (nAChRs) are targeted by alcohol and manipulations of these receptors can modify the behavioral as well as the neurotoxicological effects of alcohol (Larsson and Engel 2004; Narahashi et al., 2001; Tizabi et al, 2003, 2004b). These receptors are ligand-gated ion channels and are believed to be involved not only in drug dependency but also in diverse functions such as cognitive functions including attentional processes, learning and memory, and synaptic plasticity and neuroprotection. Various nAChR subtypes with distinct distribution and function have been identified. These receptors are composed of pentamer subunits, which can combine to form heteromeric (e.g., $\alpha 4-\beta 2, \alpha 3-\beta 2^{*}, \alpha 6^{*}$ ) or homomeric (e.g., $\alpha 7$ ) subtypes. Of these, the high-affinity nicotinic receptor $(\alpha 4-\beta 2)$ or the low-affinity subtype $(\alpha 7)$ are most abundant and widely distributed in the brain. Recent data indicate that the subunit containing $\alpha 3$ or $\alpha 6$ may be most directly involved in alcohol activation of the mesolimbic dopamine pathway (Larsson et al., 2004).

In addition to in vivo interactions between alcohol and nicotinic receptors described above, in vitro interactions have also been amply documented. Thus, it has been demonstrated that ethanol stabilizes the open channel state of the nicotinic receptors and that fast nicotinic receptor desensitization is enhanced by ethanol $(\mathrm{Wu}$ and Miller 1994). Moreover, nAChR subtypes may be differentially modulated by ethanol (Gorbounova et al 1998). Electrophysiological studies in cultured cortical neurons suggest that nicotinic receptors are sensitive conduits for mediating the central actions of ethanol and that ethanol may increase nAChR affinity for its endogenous ligand, acetylcholine (Narahashi et al., 1999). In parallel with these in vitro studies, it has been reported that long-term ethanol treatment may affect nicotinic receptor densities in selective brain regions of rats and mice (Booker and Collins, 1997; Yoshida et al., 1982). We have also reported that inherent preference for alcohol may be influenced by differential expression of striatal nicotinic receptors (Tizabi et al., 2001). Moreover, in drug discrimination paradigms, nicotine may substitute for ethanol in alcoholpreferring rats (Mcmillan et al., 1999).

Pharmacological interactions between alcohol and nicotine are further manifested in cross-tolerance observations as well as metabolic influences (Collins et al., 1996; Howard et al., 2003a, 2003b). As stated above, counteraction of the adverse effects of 1 drug by another could be a strong factor in combining the 2 drugs together. In this regard, alcohol-induced motor incoordination may be overcome by central administration of nicotine (Dar 
et al., 1994). Alcohol may also interact with nicotinic receptors to induce cognitive impairments. Nicotine, conversely, may overcome the adverse effects of alcohol via selective activation of nicotinic receptors (Wehner et al., 2004). We have observed that alcohol-induced toxicity in primary cortical and cerebellar granule cells may be blocked by nicotine pretreatment, suggesting a neuroprotective role for nicotine (Tizabi et al., 2003, 2004b). Interestingly, both high-affinity and low-affinity nicotinic receptors appear to be involved in this effect of nicotine. Blockade or attenuation of ethanol effects by nicotine or nicotinic agonists is also evident in several other in vitro studies using hippocampal slices or neuron-like pheochromocytoma (PC12) (Li et al., 2002; Prendergast et al., 2000). Considerable research has been dedicated to elucidation of the central site of nicotine effect as well as the specific nicotinic receptor subtype mediating the effect of nicotine with the expectation that such information would result in novel pharmacotherapies for drug addiction and/or neuropsychiatric or neurological disorders.

It is very important to note that the effects of smoking have to be distinguished from the effects of nicotine alone. Hence, whereas nicotine may act as a neuroprotective agent, other compounds in cigarette smoke may have quite the opposite effect. It is also conceivable that under specific conditions, nicotine may exacerbate or exaggerate some of the adverse effects of alcohol. Thus, the higher incidence of cancers in individuals with a history of heavy drinking and smoking may be aided by nicotine's ability to bioactivate tobacco-derived procarcinogens. This contention is based on the observation that cytochrome P450 $2 \mathrm{E} 1$, which can activate procarcinogens, can be induced by both alcohol and nicotine (Howard et al., 2003a, 2003b).

In summary, genetic factors and pharmacological interactions between alcohol and nicotine may be critical determining factors in comorbid conditions of drinking and smoking. Further elucidation of the neurobiological substrates of these interactions could lead to novel interventions in alcoholism, smoking, or their combination.

Acknowledgments. NIH P20 AA014643, NIH 2SO6 GM08016-33.

\section{CORTICAL BENZODIAZEPINE RECEPTORS IN ALCOHOL-DEPENDENT SMOKERS VERSUS NONSMOKERS IN SHORT-TERM SOBRIETY}

\section{Julie K. Staley, Graeme Mason, Ismene Petrakis, and John H. Krystal}

Smoking and alcoholism are synergistically associated in the general population such that persons who drink and smoke drink more than nonsmokers and drinkers smoke more than nondrinkers (Abrams et al., 1992; Bien and Burge, 1990; DiFranza and Guerrera, 1990; Gulliver et al., 1995; Johnson and Jennison, 1992). Alcohol-dependent smokers have an earlier age of onset of alcohol dependence and more alcohol-related problems (Deappen et al., 2000).
Importantly, smokers feel less intoxicated upon alcohol challenge, suggesting that smoking enhances tolerance to alcohol (Madden et al., 1995). While a central role for aberrant GABAergic function in alcohol dependence has been appreciated for decades, the role of GABA systems in cigarette smoking has been poorly studied. The paucity of studies on the regulation of GABA systems by tobacco smoke is shocking, given the high rate of comorbidity between alcoholism and tobacco smoking. Alcohol, like BZs, the primary treatment for alcohol withdrawal symptoms, facilitates GABA function. In contrast, cigarette smoke contains $\beta$ carbolines that block the actions of $\mathrm{BZ}$ at $\mathrm{GABA}_{\mathrm{A}}$ receptors, suggesting that tobacco smoking opposes the effects of alcohol at the GABA receptor and facilitates tolerance to the effects of alcohol. Initial studies in alcohol-dependent individuals abstinent for $34 \pm 20$ days demonstrated that GABA levels were decreased in the occipital cortex (Behar et al., 1999). Preliminary evidence of low cortical GABA levels in localized ${ }^{1} \mathrm{H}-\mathrm{MR}$ spectra of alcohol-dependent and hepatic encephalopathy patients and in alcohol-dependent participants abstinent for 1 to 6 months shows that BZ$\mathrm{GABA}_{\mathrm{A}}$ receptor availability was decreased in the frontal, anterior cingulate, occipital, and cerebellar cortices (AbiDargham et al., 1998) compared with healthy participants. These preliminary findings suggest that cortical GABA function is decreased over extended abstinence.

In the present studies, we sought to determine whether these GABAergic deficits also marked the early stages of recovery from alcohol dependence or whether they were solely expressed after an extended period of abstinence and whether smoking influenced the adaptive changes in $\mathrm{GABA}_{\mathrm{A}}$-receptor and GABA levels and the severity of alcohol withdrawal during early sobriety. Specifically, alcohol-dependent participants who entered the study became inpatients for 1 month. During this time, they participated in $2\left[{ }^{123}\right.$ I] iomazenil SPECT scans to measure BZ-GABA receptor levels and 2 magnetic resonance imaging (MRI) scans, performed during the first week of abstinence $(<7$ days) and repeated at 4 weeks of abstinence. To assess the effects of smoking, alcohol-dependent participants were classified by smoking status so that at $<7$ days abstinence, we studied 8 alcohol-dependent nonsmokers $(39.9 \pm 8.6$ years) and 15 alcohol-dependent smokers (40.6 \pm 8.1 years), and at 1 month of sobriety we studied 6 alcohol-dependent nonsmokers (40.8 \pm 9.9 years) and 14 alcohol-dependent smokers (38.4 \pm 7.0 years). These 4 groups of alcohol-dependent participants were compared with a single group of 15 control participants who included 10 nonsmokers $(35.9 \pm 7.5)$ and 5 smokers $(41.2 \pm 9.2)$ (Staley et al., 2005). Alcohol-dependent smokers and nonsmokers were similar in most clinical variables of alcohol drinking including age of onset, family history of smoking, severity of alcohol dependence as determined by the Alcohol Dependence Scale, and severity of craving for alcohol as measured using the Tiffany Scale for Alcohol Craving. Although the repeated time-line followback suggested that 
alcohol-dependent smokers tended to have drunk more alcohol units in the past month $(490.7 \pm 170.4$ and $444.3 \pm 194.6$ at $<7$ days and 4 weeks withdrawal, respectively) compared with alcohol-dependent nonsmokers $(340.6 \pm 148.7$ and $381.2 \pm 135.6$ at $<7$ days and 4 weeks withdrawal, respectively), the differences were not statistically significant.

Comparisons of cortical $\left[{ }^{123}\right.$ I] iomazenil uptake (determined by the outcome measure $V_{\mathrm{T}}$ [regional activity/free plasma parent], which correspond to the binding potential or $\left[B_{\max } / K_{\mathrm{D}}\right]$ ) between these 5 groups (Fig. 1), suggested that $\mathrm{GABA}_{\mathrm{A}}-\mathrm{BZ}$ receptors were higher in the frontal cortex (Brodmann's area 47), parietal cortex (Brodman's area 40), insular cortex (Brodmann's area 13), temporal cortex (Brodmann's area 21), and occipital cortex (Brodmann's area 17,19) of alcohol-dependent nonsmokers imaged within $4.6 \pm 1.8$ days from their last alcohol drink, compared with controls ( $p<0.05$ corrected for multiple comparisons). Alcohol-dependent smokers imaged within $5.0 \pm 2.9$ days were not different from controls. After 4 weeks of sobriety from alcohol, cortical $\left[{ }^{123}\right.$ I] iomazenil uptake was not significantly altered in alcohol-dependent nonsmokers or smokers compared with controls, suggesting that the GA$\mathrm{BA}_{\mathrm{A}}-\mathrm{BZ}$ receptor normalizes over the first month of abstinence from alcohol in alcohol-dependent nonsmokers.

$\left[{ }^{123} \mathrm{I}\right]$ Iomazenil uptake during the first week of sobriety correlated positively with the days since last drink in alcohol-dependent nonsmokers, suggesting that the higher iomazenil uptake reflected a compensatory increase in $\mathrm{GABA}_{\mathrm{A}}-\mathrm{BZ}$ receptors during acute alcohol withdrawal. The lack of higher iomazenil uptake combined with the lack of a correlation between iomazenil uptake and days since last alcohol drink in alcohol-dependent smokers suggests that smoking suppressed the compensatory adaptive increase in $\mathrm{GABA}_{\mathrm{A}}-\mathrm{BZ}$ receptors during acute alcohol withdrawal. Iomazenil uptake in the occipital cortex (Brodmann areas 17, 18, and 19) and the cerebellum at $<7$ days of abstinence correlated with the severity of alcohol withdrawal ( $p=0.01$ corrected for multiple comparisons) in the combined group of alcohol-dependent smokers and nonsmokers. This suggests that $\mathrm{GABA}_{\mathrm{A}}-\mathrm{BZ}$ receptor availability may predict the severity of alcohol withdrawal. Given that in this sample, the alcohol-dependent nonsmokers tended to demonstrate more severe alcohol withdrawal (peak CIWA scores of $6.9 \pm 4.8$; mean \pm SD) compared with alcohol-dependent smokers (peak CIWA $4.5 \pm 2.9$ mean $\pm \mathrm{SD}$ ), these findings imply that by suppressing the compensatory increase in $\mathrm{GABA}_{\mathrm{A}}$-BZ receptors, smoking may suppress alcohol withdrawal symptoms.

In an overlapping sample of participants, occipital GABA levels were measured using magnetic resonance spectroscopy (MRS). This study population included 12 alcohol-dependent individuals (5 nonsmokers and 7 smokers) and 8 control individuals (4 nonsmokers and 4 smokers) (Mason et al., 2005). A tendency toward higher cortical GABA levels was noted in alcohol-dependent nonsmokers at 5 days of abstinence compared with control
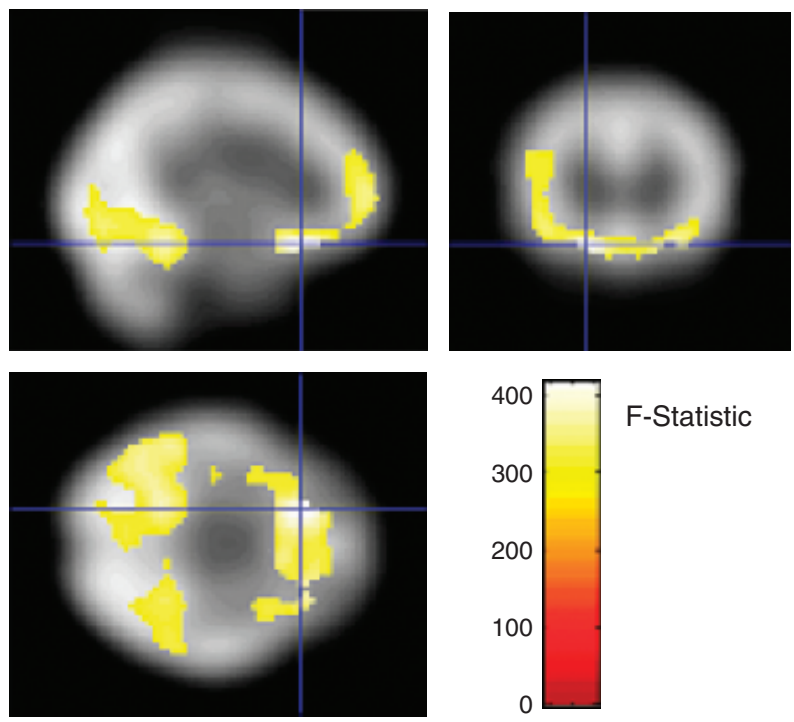

Fitted and adjusted responses all effects

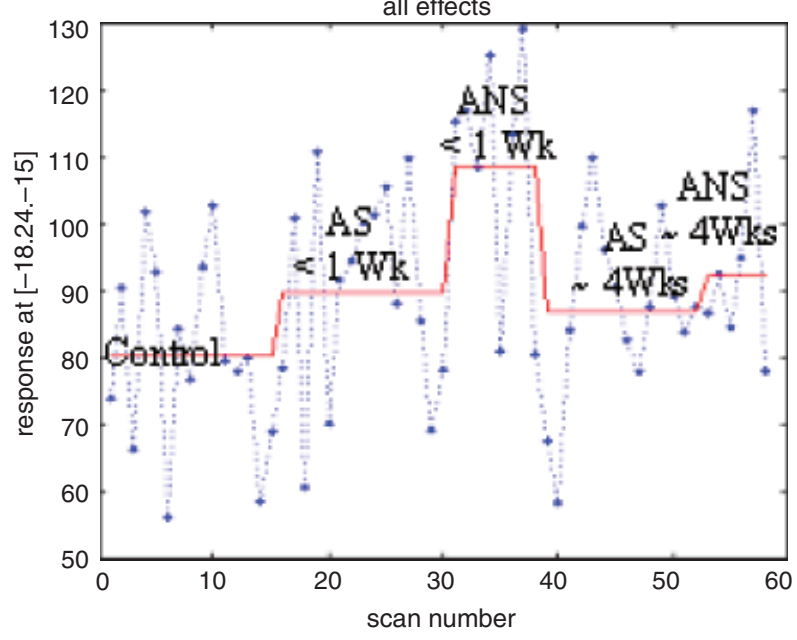

Fig. 1. $\left[{ }^{123}\right.$ I] lomazenil binding to brain $G A B A_{A}-B Z$ receptors in alcoholic smokers and nonsmokers. Top: Brain areas that are significantly different $\left(F_{5,53}>300\right)$ for comparisons of $\left[{ }^{123} \mid\right]$ iomazenil uptake (VT) between 5 groups including: Group 1, healthy controls $(n=15)$; Group 2, alcoholdependent smokers $<1$ week abstinence $(n=15)$; Group 3, alcohol-dependent nonsmokers $<1$ week abstinence $(n=8)$; Group 4, alcohol-dependent smokers $\sim 4$ weeks abstinence $(n=14)$; Group 5 , alcohol-dependent nonsmokers $\sim 4$ weeks abstinence $(n=6)$. Bottom: Plot of the activity at the voxel with the highest $F$ value in (A). Dots represent actual VT values for individual participants in each group; the mean values for each of the 5 groups are represented by the line. These findings suggests that the alcohol-dependent nonsmokers (ANS $<1$ week) have higher uptake at $\sim 1$ week of abstinence compared with controls and alcohol-dependent smokers ( $\mathrm{aS}<1$ week and $\mathrm{AS} \sim 4$ weeks). BZ, benzodiazepine, GABA, $\gamma$-aminobutyric acid.

individuals $(p=0.09)$, and cortical GABA levels were significantly higher in alcohol-dependent nonsmokers compared with alcohol-dependent smokers at 5 days of sobriety $(p=0.004)$. At 4 weeks of sobriety, GABA levels in nonsmokers had normalized to the same level as in alcohol-dependent smokers and they were not significantly different from levels in control.

Collectively, the findings suggest that the severity of alcohol withdrawal may be determined in part by $\mathrm{GABA}_{\mathrm{A}}$ 
receptor availability and that withdrawal severity is suppressed by cigarette smoking. Furthermore, these data demonstrate time-dependent regulation of cortical GABA and $\mathrm{GABA}_{\mathrm{A}}-\mathrm{BZ}$ receptors associated with the recovery from alcohol dependence. Higher $\mathrm{GABA}_{\mathrm{A}}-\mathrm{BZ}$ receptor levels may reflect subunit changes and decreased $\mathrm{GABA}_{\mathrm{A}^{-}}$ $\mathrm{BZ}$ receptor function induced by protracted alcohol drinking. Higher GABA levels during acute withdrawal may reflect a compensation for reduced receptor function, thought to contribute to alcohol tolerance and withdrawal, whereas the subsequent decline may reflect "normalization" of $\mathrm{GABA}_{\mathrm{A}}$ receptor function with sobriety.

Smoking may attenuate $\mathrm{GABA}_{\mathrm{A}}$ receptor adaptations associated with alcohol dependence and may contribute to the comorbidity between alcoholism and smoking. Given that BZs are usually the first line of treatment for alcohol withdrawal symptoms, these findings suggest that BZs may be differentially effective in alcohol-dependent individuals who smoke versus those who do not.

Acknowledgments. NIH K01AA00288, NIH K05 AA014715-01, NIH RO1 AA1132, NIH P50 DA13334; the Dana Foundation; the Mental Illness Research, Education, and Clinical Center for Dual Diagnosis (MIRE$\mathrm{CC})$; and the VA Alcohol Research Center.

\section{BRAIN STRUCTURE, METABOLITES, AND PERFUSION IN ALCOHOLIC SMOKERS AND NONSMOKERS DURING SOBRIETY}

\section{Timothy C. Durazzo, Stefan Gazdzinski, Peter Banys, and Dieter J. Meyerhoff}

Tobacco products (mainly cigarettes) are the most commonly used substances among actively drinking and recovering alcoholic patients. Increasing evidence from neuroimaging studies links chronic smoking to adverse effects on brain morphology, cerebral blood flow, and neurochemistry independent of excessive alcohol consumption. Compared with nonsmokers, chronic smokers demonstrated lower cortical gray matter volumes and densities in the prefrontal cortex, smaller left anterior cingulate volume, and lower gray matter densities in the right cerebellum (Brody et al., 2004), as well as increased generalized brain atrophy with advancing age (Hayee et al., 2003). Active smokers showed lower global cerebral blood flow and limbic system blood flow (Domino et al., 2004) than nonsmokers. It appears that chronic cigarette smoking has some of the same adverse effects on the human brain as chronic alcoholism; however, the potential neurobiologic consequences of chronic cigarette smoking were not considered in past neuroimaging studies of individuals with AUD. Thus, it is unclear whether the full extent of the neurobiological abnormalities reported in neuroimaging studies with alcoholic individuals are solely related to chronic, excessive alcohol consumption or whether concurrent chronic cigarette smoking may contribute to the aberrations observed.
In 1-week abstinent recovering alcoholic patients (RA), we investigated brain morphology with high-resolution MRI (Gazdzinski et al., 2005), brain metabolite concentrations with proton magnetic resonance spectroscopic imaging $\left({ }^{1} \mathrm{H}\right.$ MRSI) (Durazzo et al., 2004), and brain perfusion with pulsed arterial spin labeling. RA were again studied with ${ }^{1} \mathrm{H}$ MRSI after 1 month of abstinence. To investigate the potential independent and combined effects of concurrent chronic cigarette smoking and alcohol dependence, we retrospectively divided our RA cohort into smokers and nonsmokers. All data were acquired on a clinical 1.5-T MR scanner.

\section{Cross-sectional MR Studies in 1-week Abstinent Recovering Alcoholic Patients}

High-resolution 3-dimensional MRIs were acquired from 1-week abstinent nonsmoking RA (nsRA; $n=13$; age $49.8 \pm 9.7$ years) and smoking recovering alcoholic patients (sRA; $n=24$; age $49.4 \pm 8.3$ years) as well as from light drinking (LD) nonsmokers (nsLD; $n=7$; age $47.5 \pm 6.0$ years) and smokers (sLD; $n=23$; age $38.1 \pm 8.7$ years). All RA met Diagnostic and Statistical Manual-fourth edition (DSM-IV) criteria for alcohol dependence with physiological dependence. Smoking recovering alcoholic individuals consumed $290 \pm 120$, and nsRA $200 \pm 120$ drinks per month over a lifetime. Smoking recovering alcoholic individuals smoked $21 \pm 8$ cigarettes per day for $23 \pm 13$ years; cigarette pack-years was $26 \pm 19$ and their Fagerstrom score was $5.5 \pm 1.9$. Eightyseven percent of sLD participants smoked daily or nearly every day. A brief neurocognitive battery assessed visuospatial learning and memory, working memory, and visuomotor scanning and incidental learning. Regional white matter (WM), gray matter (GM), and CSF volumetry, corrected for intracranial volume, used automated probabilistic segmentation, combined with automated atlasbased region labeling of the major lobes, cerebellum, and subcortical structures (Fig. 2). Regional brain volumes were converted into age-corrected $z$ scores based on nsLD. Multivariate analysis of variance for all measured regions yielded main effects for both alcohol and smoking. Follow-up ANOVAs demonstrated alcohol main effects for the parietal and temporal GM, with less GM in RA than LD. Significant smoking effects were found for parietal, temporal, and occipital GM, where smokers demonstrated less GM in these regions than nonsmokers (Fig. 3). Alcohol main effects were also seen for the frontal WM and parietal WM, with smaller WM in RA compared with LD. A large smoking main effect for the temporal WM and a trend for frontal WM were observed, with larger WM volumes in smokers versus nonsmokers. In nsRA, visuospatial learning and memory positively correlated with temporal WM and occipital WM volumes. No significant structure-neurocognitive relationships were noted for sRA. The larger temporal and frontal WM volumes in 

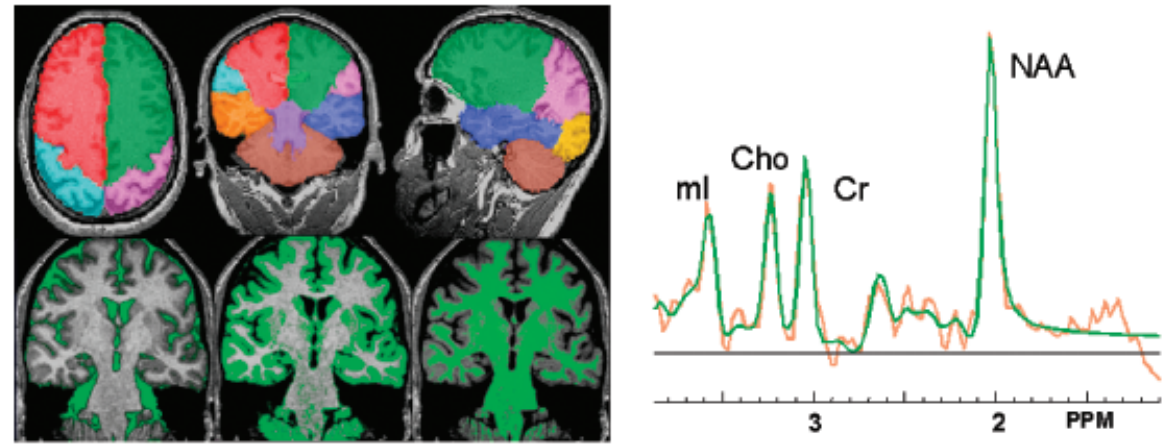

Fig. 2. Left: Top images-identification of major anatomical subdivisions using atlas-based methods; bottom images-probabilistic segmentation of highresolution T1-weighted magnetic resonance images. The images show probabilities for cerebrospinal fluid, gray matter, and white matter, where higher probabilities of correct tissue classification are reflected in brighter color. Right: Example of a single metabolite spectrum obtained from 1 of the 3 spectroscopic imaging planes. The orange line represents the experimental spectrum and the green line shows the fitted spectrum. ml, myoinositol; Cho, choline-containing compounds; $\mathrm{Cr}$, creatine and phosphocreatine; $\mathrm{NAA}=\mathrm{N}$-acetylaspartate.

sRA may be associated with cytotoxic and/or vasogenic swelling secondary to smoking-induced alterations in mitochondrial function and vascular endothelial damage, respectively. It has been suggested that late-myelinating areas such as the frontal and temporal lobes may be particularly vulnerable to increased oxidative stress and cerebral hypoperfusion (see Bartzokis, 2004a, 2004b), which are both found in chronic smokers. In general, findings of GM loss are functionally significant, as brain shrinkage is a risk factor for cognitive decline and memory impairment in the elderly.

Short-echo-time multislice ${ }^{1} \mathrm{H}$ MRSI was performed on 14 nsRA and 10 sRA. RA participant demographics, smoking characteristics, and neurocognitive measures administered were similar to those reported for the brain morphology study. Concentrations of $\mathrm{N}$-acetylaspartate (NAA), choline-containing compounds (Cho), and other metabolites were derived from spectra (see Fig. 2 for an example spectrum) measured in 3 parallel planes through the centrum semiovale, basal ganglia, and cerebellar vermis. Regional atrophy-corrected metabolite concentrations were

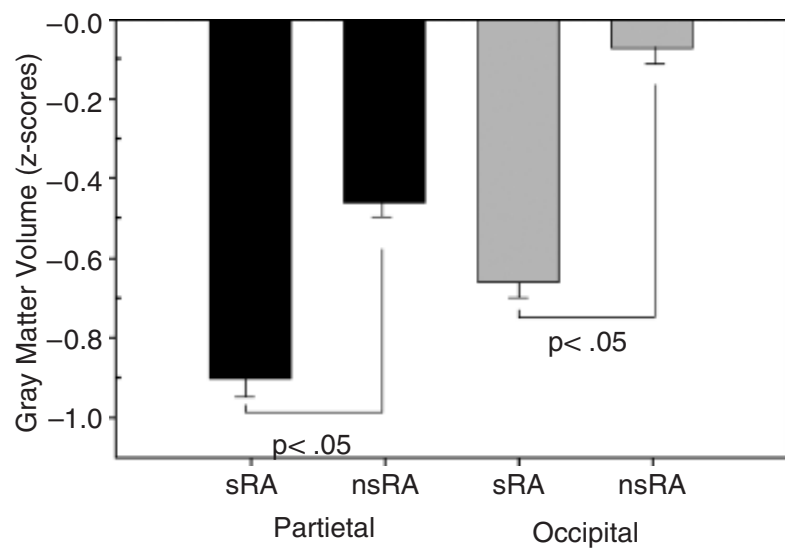

Fig. 3. Comparison of parietal and occipital gray matter volumes among 1-week abstinent smoking recovering alcoholic individuals (sRA) and nonsmoking recovering alcoholic individuals (nsRA). Larger negative $z$ scores reflect greater volume loss relative to nonsmoking light drinkers (mean and SE). i.u., institutional units. calculated by combining ${ }^{1} \mathrm{H}$ MRSI and segmented MRI data. Smoking recovering alcoholic individuals compared with nsRA demonstrated $10 \%$ lower NAA in the frontal WM and $15 \%$ lower NAA and $21 \%$ lower Cho in the midbrain (Fig. 4). In addition, sRA showed trends to lower NAA in the parietal GM and lenticular nuclei. Results were unchanged after controlling for nonsignificantly higher average number of drinks per month over a lifetime in sRA. In sRA, higher nicotine dependence and number of cigarettes smoked per day were negatively correlated with thalamic and lenticular NAA levels. In nsRA, cerebellar vermis NAA was positively related to visuospatial learning and visuospatial memory, whereas in sRA, cerebellar vermis NAA was positively related to visuomotor scanning speed and incidental learning. Lower brain metabolite levels in sRA may be related to the additional direct and indirect biologic consequences associated with chronic cigarette smoking such as decreased cerebral perfusion, hypoxia, and increased oxidative stress.

Perfusion-weighted images (PWI) were acquired with a new pulsed arterial spin labeling method (Jahng et al., 2003) from 36 one-week abstinent RA (10 nsRA and 19 sRA) and 19 nsLD (Gazdzinski et al., 2006). Demographics and smoking characteristics were similar to those reported for the brain morphology study. Multivariate analysis of variance on frontal and parietal GM perfusion for all 3 groups demonstrated a group effect where sRA showed lower frontal GM perfusion (Fig. 5) and parietal GM perfusion than both nsLD and nsRA. Gray matter perfusion was similar in nsRA and nsLD. Parietal GM perfusion in sRA correlated inversely with the number of cigarettes smoked per day, but it did not correlate with recency of last cigarette smoked. This suggests that chronic and not acute effects of cigarette smoking modulate brain perfusion in RA.

\section{Longitudinal ${ }^{1}$ H MRSI Studies over 1 Month of Abstinence from Alcohol}

Short-echo time multislice ${ }^{1} \mathrm{H}$ MRSI was performed with $11 \mathrm{nsRA}$ and 14 sRA $6 \pm 3$ days after consumption 

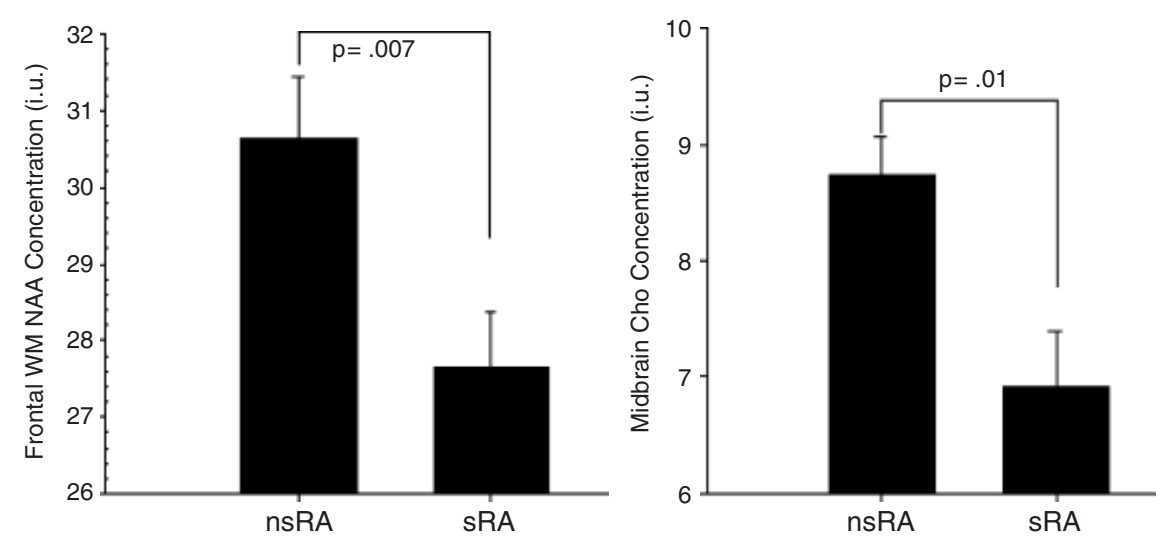

Fig. 4. Comparison of frontal $\mathrm{N}$-acetylaspartate (NAA) (left) and midbrain Cho concentrations (right) among 1-week abstinent smoking recovering alcoholic individuals (sRA) and nonsmoking recovering alcoholic individuals (nsRA). Higher values represent greater metabolite concentration (mean and SE). i.u., institutional units.

of their last alcoholic drink and again after $34 \pm 10$ days of abstinence. Demographics and smoking characteristics were similar to those reported for the brain morphology study. The nsRA group showed an increase of frontal WM NAA and a trend toward increasing frontal GM NAA over 1 month of abstinence. Nonsmoking recovering alcoholic individuals demonstrated significant Cho increases in the GM of the frontal, parietal, and temporal lobes, as well as Cho increases in the WM of the frontal, parietal, temporal, and occipital lobes. In the sRA group, NAA concentrations significantly increased only in the frontal GM, while NAA decreased in both the parietal WM and the occipital WM over 1 month of abstinence from alcohol. Smoking recovering alcoholic individuals demonstrated Cho increases only in the frontal lobe. The localized and rather poor recovery of Cho in the sRA cohort during short-term abstinence from alcohol may be related to continued exposure to the numerous sources of oxidative stress in cigarette smoke.

These preliminary MR studies in abstinent RA suggest the following: (1) chronic alcohol dependence and cigarette smoking both promote volume loss in cortical GM,

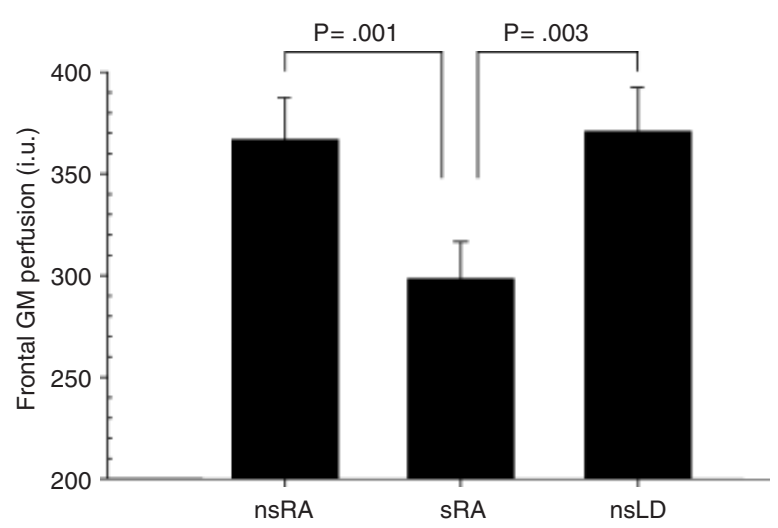

Fig. 5. Comparison of frontal gray matter perfusion among nonsmoking light drinkers and 1-week abstinent smoking recovering alcoholic individuals (sRA) and nonsmoking recovering alcoholic individuals (nsRA). Higher values represent greater perfusion (mean and SE). i.u., institutional units. and smoking is associated with (pathological?) increases in temporal WM volume; (2) cigarette smoking compounds ${ }^{1} \mathrm{H}$ MRSI are markers of chronic alcohol-induced neuronal injury and cell membrane damage in the frontal lobes and the midbrain of 1-week abstinent RA in a dosedependent manner; (3) chronic cigarette smoking compounds alcohol-induced perfusion deficits in the frontal and parietal GM of 1-week abstinent RA; (4) chronic cigarette smoking adversely affects brain metabolite recovery during short-term abstinence from alcohol (up to 1 month); and (5) chronic smoking in RA may further compromise alcohol-induced disturbances in functional neurocircuitry (Sullivan and Pfefferbaum, 2005) thereby modulating relationships between MR-derived neurobiologic measures and neurocognition; therefore, examining RA as a single group irrespective of smoking status may obscure the ability of MR-derived neurobiologic measures to serve as useful surrogate markers of brain function.

Overall, our preliminary results suggest that the effects of concurrent chronic cigarette smoking should be considered in future studies investigating the consequences of AUD on brain structure, function, metabolism, and their recovery during abstinence, as well as in research of other neuropsychiatric conditions (e.g., bipolar disorder, schizophrenia) in which cigarette smoking is highly comorbid.

Acknowledgment. NIH R01 AA10788 (Meyerhoff)

\section{NEUROCOGNITIVE FUNCTION, SMOKING, AND ALCOHOLISM}

Jennifer M. Glass, Kenneth M. Adams, Joel T. Nigg, Maria M. Wong, Leon I. Puttler, Anne Buu, Jennifer M. Jester, Hiram E. Fitzgerald, and Robert A. Zucker

A substantial literature documents neurocognitive deficits in severe, chronic alcoholic men (Knight and Longmore, 1994). The observed impairments include difficulties with visual-spatial processing, problem solving, memory, and cognitive proficiency (Nixon et al., 1995, 1998) .Variability across studies in both the magnitude and the type 
of neurocognitive deficits observed points to multiple paths to brain and cognitive dysfunction among alcoholic individuals (Adams and Grant, 1986; Tarter and Edwards, 1986), including comorbid psychological and medical conditions secondary to alcoholism. Additionally, abuse of other drugs is common among alcoholic populations and is a factor that is addressed by researchers as it is expected to affect neurocognitive function (Nixon et al., 1998). However, one important and commonly used other drug that has received little attention on neurocognitive function in alcoholism is nicotine/cigarette smoking. Estimates of cigarette smoking among alcoholic individuals range from $50 \%$ to $80 \%$, compared with $23 \%$ in the general population in the United States (see Introduction); yet it is generally not mentioned in studies of neurocognitive function in alcoholism. This is a potentially important gap in understanding, because chronic cigarette smoking is linked to poor cognitive performance.

Cigarette smoking has 2 potentially different effects on neurocognitive performance, an acute effect of nicotine and a chronic effect because of long-term smoking (e.g., vascular disease and chronic obstructive pulmonary disease). Acute administration of nicotine leads to some improvement in cognitive performance, especially on tasks of sustained attention or vigilance (Ernst et al., 2001). This has even been demonstrated in alcoholic patients (Craddock et al., 2003). Conversely, chronic cigarette smoking is associated with cognitive impairment in several epidemiological studies. For example, smokers performed more poorly than both never-smokers and ex-smokers on tests of general cognitive function (Galanis et al., 2000), working memory (Ernst et al., 2001), psychomotor speed (Hill, 1989; Kalmijn et al., 2002), and cognitive flexibility (Kalmijn et al., 2002). In sum, although there are beneficial effects from nicotine on cognitive performance, the longterm effects of smoking are clearly negative and seem most pronounced on measures that emphasize the ability to rapidly and flexibly process information.

We hypothesized that smoking may be an important part of the etiology of neurocognitive impairment in alcoholic individuals. Because smoking is more prevalent among alcoholic individuals, previous studies may have been biased and differences in neurocognitive function may have been attributed to alcoholism that were in fact associated with the different rates of smoking in the alcoholic and control groups.

We examined this hypothesis (Glass et al., 2005) in a community-recruited sample $(n=172)$ of alcoholic men and controls who were taking part in a longitudinal family study of risk factors for substance abuse in children (Zucker et al., 1996). Neurocognitive function was measured with intelligence quotient (IQ) from the short version of the Wechsler Adult Intelligence Scale-Revised (Sattler, 2001) and cognitive proficiency from the Microcog Assessment of Cognitive Functioning (Powell et al., 1994). Microcog assesses 6 distinct aspects of cognitive function (short-term memory, immediate recall, delayed recall, visual-spatial ability, verbal reasoning, and mathematical reasoning) and provides a measure of global cognitive proficiency (accuracy scaled by response time) as well as proficiency for each subtest. Proficiency has been shown to be a more sensitive measure of neurocognitive function in alcoholic individuals than accuracy alone (Nixon et al., 1995,1998 ) and information processing fluency or proficiency impairments are reported for smokers; therefore, we focused our analyses on the global proficiency measure provided by Microcog and on IQ.

Alcoholism was measured in several ways as follows: lifetime DSM-IV diagnosis, long-term average rate of drinking (past 9-12 years), short-term average rate of drinking (past 6 months), and with the Lifetime Alcohol Problems Score (LAPS). We found that LAPS was most highly and consistently correlated with the neurocognitive measures, so it became the measure of alcoholism used in subsequent analyses. LAPS is constructed of 3 subscores that assess onset, variety, and life-invasiveness of alcoholic symptoms. Smoking was measured by pack-years (average cigarette smoking measured in packs per day multiplied by years of smoking).

Both smoking and alcoholism were correlated with worse neurocognitive function; when both were included in regression analyses, smoking remained a significant predictor of both IQ and cognitive proficiency, but alcoholism was significant only for IQ (Figs. 6 and 7). This suggests that smoking mediated the effect of alcoholism for the proficiency measure, but both smoking and alcoholism are unique predictors of IQ. The interaction between smoking and alcoholism was not significant for either IQ or cognitive proficiency.

We also examined the effects of smoking and alcoholism on response inhibition using the stopping task that measures the ability to withhold a response during a reactiontime task. In $25 \%$ of trials, a tone is presented that signals the participants to stop or withhold their response. The timing of the tone is dynamically adjusted so that each participant's ability to stop a response can be measured (the Stop RT; Logan et al., 1984). A slow Stop RT is associated with impulsiveness and poor response inhibition (Nigg, 1999). Again, both smoking and alcoholism were correlated with slower Stop RTs. When included in a regression analysis, both smoking and alcoholism and the interaction were significant predictors of Stop RT (Fig. 8). This suggests that smoking and alcoholism are uniquely associated with the ability to stop a response and that smoking and alcoholism combine to predict even worse performance on the stopping task.

These results demonstrate that smoking is an important factor to consider when studying cognitive function in an alcoholic sample. It appears that for measures of fast, flexible performance, smoking may explain the effects of alcoholism, but for other measures such as IQ or response inhibition, both smoking and alcoholism are predictors of 


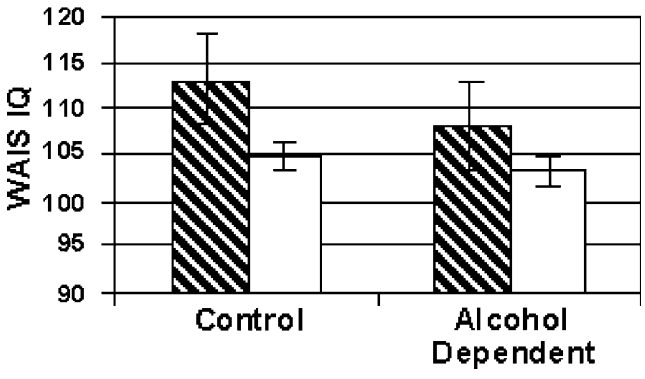

Fig. 6. Intelligence quotient, smoking, and alcoholism.

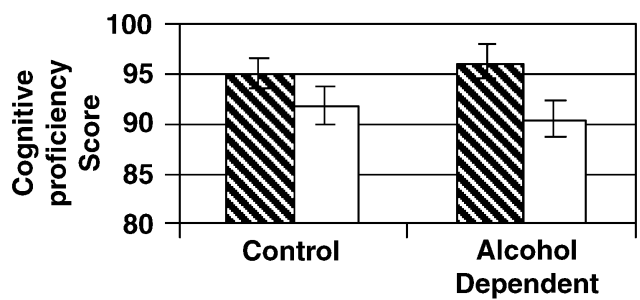

Fig. 7. Cognitive proficiency, smoking, and alcoholism.
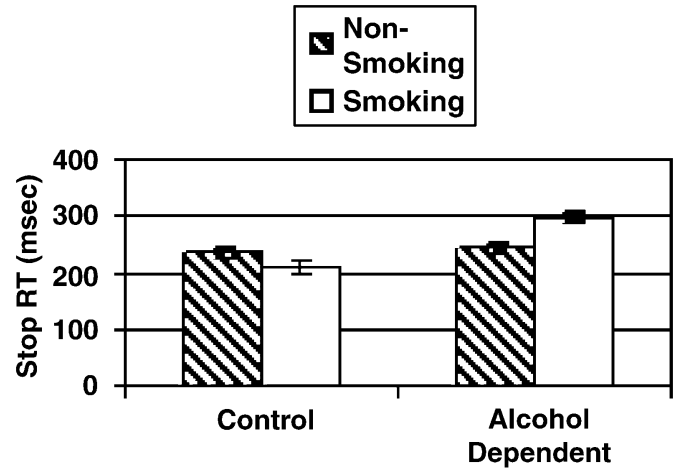

Fig. 8. Response inhibition, smoking, and alcoholism.

neurocognitive function. It is tempting to speculate that long-term effects of nicotine on brain function, or long-term effects of smoking on cerebro-vascular health may explain these results. Further study will be necessary to establish a causal mechanism and to generalize the findings to women.

Acknowledgments. NIH R337 AA07065 and NIH AA12217 (Zucker, Fitzgerald, Nigg)

\section{DISCUSSION}

\section{SaraJo Nixon}

The direct and interactive effects of nicotine on brain function have been clinically and anecdotally appreciated for some time. However, the systematic study of acute and chronic nicotine use, particularly in individuals with other substance use disorders, has received little general attention. Over the past decade, though, some concerted efforts have been directed toward addressing this paucity of research in both basic and applied applications. This symposium presented research overviews and cutting-edge

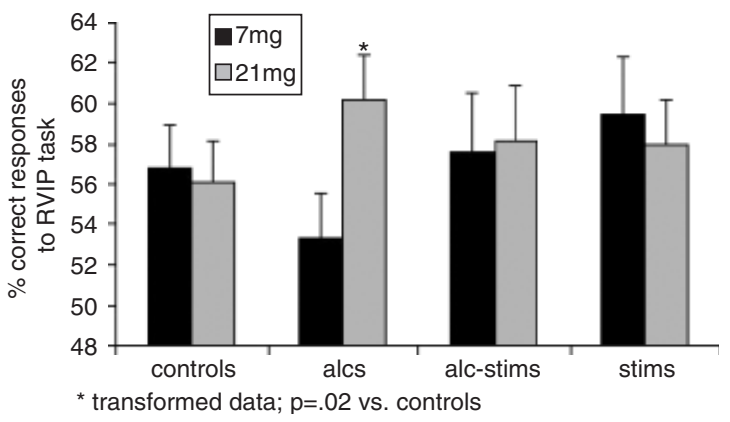

Fig. 9. Higher nicotine levels improve vigilance in alcoholic individuals (alcs). alc-stims, stimulant abusers with a history of alcohol dependence; stims, stimulant abusers without a history of alcohol dependence.

science that suggests that the neurobiologic and neurocognitive abnormalities commonly described in past studies of alcohol-dependent individuals may be at least partially modulated by concurrent chronic tobacco use. Furthermore, behavioral recovery, if not biological brain recovery in abstinent alcoholic individuals may be affected by chronic and continued smoking. This latter issue is only beginning to be addressed in dose-response studies of acute nicotine administration in otherwise abstinent substance abusers. Initial data suggest that nicotine may be differentially effective in altering attentional processes (e.g., vigilance and covert visual attention), with abstinent alcoholic individuals being more sensitive to nicotine's cognitive-enhancing effects than other substance abuse groups, such as stimulant abusers without a history of alcohol dependence (Fig. 9) (Ceballos et al., 2006).

This symposium was intended to raise awareness among fellow researchers that smoking can no longer be ignored as a potentially significant contributor to brain injury in alcoholism. Current research methodology is able to contribute to our understanding of the potential separate and interactive effects of chronic nicotine/smoking and alcohol consumption at the molecular, biological, and cognitive/ behavioral levels. Specific mechanisms of biological brain injury and recovery from alcoholism can be illuminated in humans as a function of smoking status and in connection with common and distinctly separate neurocognitive consequences of smoking. Nevertheless and despite the wellknown comorbidity, this type of research has not received sufficient attention in the past. The organizers and presenters of this symposium expect that they contributed useful information to both smoking and alcohol research that ultimately can inform ongoing discussions about treatment strategies for these often comorbid disorders, educate the public, and possibly influence health and public policy in general.

\section{ACKNOWLEDGMENTS}

NIH DA 013677 (Nixon) and University of Kentucky Office for Vice President of Research (Nixon), NIH R31 DA 06086 (Ceballos). Contributions: Rick D. Tivis, 
Natalie Ceballos, Andrea Craddock, Robert Prather, Amanda Ross.

\section{REFERENCES}

Abi-Dargham A, Krystal J, Anjivel S, Scanley E, Zoghbi S, Baldwin R, Rajeevan N, Seibyl J, Charney D, Laruelle M, Innis R (1998) Alterations of benzodiazepine receptors in type II alcoholics measured with SPECT and [123I]iomazenil. Am J Psychiatry 155:1550-1555.

Abrams D, Rohsenow D, Niaura R, Pedraza M, Longabraugh R, Beattie M, Binkoff J, Noel N, Monti P (1992) Smoking and treatment outcome for alcoholics: effects on coping skills, urge to drink, and drinking rates. Behav Ther 23:283-297.

Adams KM, Grant I (1986) Influence of premorbid risk factors on neuropsychological performance in alcoholics. J Clin Exp Neuropsychol $8: 362-370$

Bartzokis G (2004a) Age-related myelin breakdown: a developmental model of cognitive decline in Alzheimer's disease. Neurobiol Aging 25:5-18.

Bartzokis G (2004b) Quadratic trajectories of brain myelin content: unifying construct for neuropsychiatric disorders. Neurobiol Aging 25:49-62.

Behar K, Rothman D, Petersen K, Hooten M, Delaney R, Petroff O, Shulman G, Navarro V, Petrakis I, Charney D, Krystal J (1999) Preliminary evidence of low cortical GABA levels in localized 1H-MR spectra of alcohol-dependent and hepatic encephalopathy patients. Am J Psychiatry 156:952-954.

Bien T, Burge R (1990) Smoking and drinking: a review of the literature. Int J Addict 25:1429-1454.

Blomqvist O, Hernandez-Avila CA, Van Kirk J, Rose JE, Kranzler HR (2002) Mecamylamine modifies the pharmacokinetics and reinforcing effects of alcohol. Alcohol Clin Exp Res 26:326-331.

Booker TK, Collins AC (1997) Long-term ethanol treatment elicits changes in nicotinic receptor binding in only a few brain regions. Alcohol 14:131-140.

Brody AL, Mandelkern MA, Jarvik ME, Lee GS, Smith EC, Huang JC, Bota RG, Bartzokis G, London ED (2004) Differences between smokers and nonsmokers in regional gray matter volumes and densities. Biol Psychiatry 55:77-84.

Campbell VC, Taylor RE, Tizabi Y (2005) Antinociceptive effects of alcohol and nicotine in a rat model: involvement of opioid system. Alcohol Clin Exp Res 29 (suppl): 14A.

Ceballos NA, Tivis R, Lawton-Craddock A, Nixon SJ (2006) Cognitive efficiency in substance abusers: effects of nicotine replacement. Subst Use Misuse 41:1-17.

Collins AC, Wilkins LH, Slobe BS, Cao JZ, Bullock AE (1996) Longterm ethanol and nicotine treatment elicit tolerance to ethanol. Alcohol Clin Exp Res 20:990-999.

Craddock A, Cheek JA, Tivis R, Nixon SJ (2003) Nicotine's effects on neurocognitive performance in alcoholics. Alcohol Clin Exp Res 27:140A.

Currie SR, Hodgins DC, el-Guebaly N, Campbell W (2001) Influence of depression and gender on smoking expectancies and temptations in alcoholics in early recovery. J Subst Abuse 13:443-458.

Dar MS, Bowman ER, Chunxiao L (1994) Intracerebellar nicotiniccholinergic participation in the cerebellar adesoinergic modulation of ethanol-induced motor incoordination in mice. Brain Res 644:117127.

Daeppen JB, Smith TL, Danko GP, Gordon L, Landi NA, Nurnberger JL Jr, Bucholz KK, Raimo E, Schuckit MA (2000) Clinical correlates of cigarette smoking and nicotine dependence in alcohol-dependent men and women. The Collaborative Study Group on the Genetics of Alcoholism. Alcohol Alcohol 35:171-175.

DiFranza J, Guerrera M (1990) Alcoholism and smoking. J Stud Alcohol $51: 130-135$
Domino EF, Ni L, Xu Y, Koeppe RA, Guthrie S, Zubieta JK (2004) Regional cerebral blood flow and plasma nicotine after smoking tobacco cigarettes. Prog Neuropsychopharmacol Biol Psychiatry 28:319-327.

Durazzo TC, Gazdzinski S, Banys P, Meyerhoff DJ (2004) Cigarette smoking exacerbates chronic alcohol-induced brain damage: a preliminary metabolite imaging study. Alcohol Clin Exp Res 28:1849-1860.

Ericson M, Blomqvist O, Engel JA, Soderpalm B (1998) Voluntary ethanol intake in the rat and the associated accumbal dopamine overflow are blocked by ventral tegmental mecamylamine. Eur J Pharmacol 358:189-196.

Ernst M, Heishman SJ, Spurgeon L, London ED (2001) Smoking history and nicotine effects on cognitive performance. Neuropsychopharmacology 25:313-319.

Galanis DJ, Joseph C, Masaki KH, Petrovitch H, Ross GW, White L (2000) A longitudinal study of drinking and cognitive performance in elderly Japanese American men: the Honolulu-Asia Aging Study. Am J Public Health 90:1254-1259.

Gazdzinski S, Durazzo TC, Meyerhoff DJ (2005) Temporal dynamics and determinants of whole brain tissue volume changes during recovery from alcohol dependence. Drug Alcohol Depend 78:263-273.

Gazdzinski S, Durazzo TC, Jahng GH, Ezekiel F, Banys P, Meyerhoff DJ (2006) Effects of chronic alcohol dependence and chronic cigarette smoking on cerebral perfusion - a preliminary magnetic resonance study. Alcohol Clin Exp Res 30: in press.

Glass JM, Adams KM, Nigg JT, Wong MM, Puttler LI, Buu A, et al (2005) Smoking is associated with neurocognitive deficits in alcoholism. Drug Alcohol Depend [Epub ahead of print].

Gorbounova O, Svensson AL, Jonsson P, Mousavi M, Miao H, Hellstrom-Lindahl E, Nordberg A (1998) Chronic ethanol treatment decreases $\left[{ }^{3} \mathrm{H}\right]$ epibatidine and $\left[{ }^{3} \mathrm{H}\right]$ nicotine binding and differentially regulates mRNA levels of nicotinic acetylcholine receptors subunits expressed in M10 and SH-SY5Y neuroblastoma cells. J Neurochem 70:1134-1142.

Gulliver S, Rohsennow D, Colby S, Dey A, Abrams D, Niaura R, Monti $P$ (1995) Interrelationship of smoking and alcohol dependence, use and urges to use. J Stud Alcohol 56:202-206.

Hayee A, Haque A, Anwarullah A, Rabbani M (2003) Smoking enhances age related brain atrophy-a quantitative study with computed tomography. Bangladesh Med Res Council Bull 29:118-124.

Hill RD (1989) Residual effects of cigarette smoking on cognitive performance in normal aging. Psychol Aging 4:251-254.

Howard LA, Ahluwalia JS, Lin SK, Sellers EM, Tyndale RF (2003a) CYP2E1*1D regulatory polymorphism: association with alcohol and nicotine dependence. Pharmacogenetics 13:321-328 (Erratum in: Pharmacogenetics. 2003 Jul;13(7):441-2).

Howard LA, Miksys S, Hoffmann E, Mash D, Tyndale RF (2003b) Brain CYP2E1 is induced by nicotine and ethanol in rat and is higher in smokers and alcoholics. Br J Pharmacol 138:1376-1386.

Hurt RD, Eberman KM, Croghan IT, Offord KP, Davis LJ Jr, Morse RM, Palmen MA, Bruce BK (1994) Nicotine dependence treatment during inpatient treatment for other addictions: a prospective intervention trial. Alcohol Clin Exp Res 18:867-872.

Hurt RD, Offord KP, Croghan IT, Gomez-Dahl L, Kottke TE, Morse RM, Melton LJ III (1996) Mortality following inpatient addictions treatment. Role of tobacco use in a community-based cohort. JAMA 275:1097-1103.

Jahng GH, Zhu XP, Matson GB, Weiner MW, Schuff N (2003) Improved perfusion-weighted MRI by a novel double inversion with proximal labeling of both tagged and control acquisitions. Magn Reson Med 2003 49:307-314.

Johnson K, Jennison K (1992) The drinking-smoking syndrome and social context. Int J Addiction 27:749-792.

Kalmijn S, van Boxtel MP, Verschuren MW, Jolles J, Launer LJ (2002) Cigarette smoking and alcohol consumption in relation to cognitive performance in middle age. Am J Epidemiol 156:936-944. 
Knight RG, Longmore BE (1994) Clinical Neuropsychology of Alcoholism. Lawrence Erlbaum Associates Ltd, Hove, UK.

Larsson A, Engel JA (2004) Neurochemical and behavioral studies on ethanol and nicotine interactions. Neurosci Biobehav Rev 27:713-720.

Larsson A, Jerhlag E, Svensson L, Soderpalm B, Engel JA (2004) Is an a-conotoxin MII-sensitive mechanism involved in the neurochemical, stimulatory, and rewarding effects of ethanol? Alcohol 34:239-250.

Lê AD, Corrigall WA, Watchus J, Harding S, Juzytsch W, Li TK (2000) Involvement of nicotinic receptors in alcohol self-administration. Alcohol Clin Exp Res 24:155-163.

Lê AD, Wang A, Harding S, Juzytsch W, Shaham Y (2003) Nicotine increases alcohol self-administration and reinstates alcohol seeking in rats. Psychopharmacology (Berlin) 168:216-221.

Li XC, Karandash MS, Jenkins PM, Stitzel JA (2005) Genetic correlation between the free-choice oral consumption of nicotine and alcohol in $\mathrm{C} 57 \mathrm{BL} / 6 \mathrm{~J} \times \mathrm{C} 3 \mathrm{~h} / \mathrm{HeJ} \mathrm{F} 2$ intercross mice. Behav Brain Res 157:7990.

Li Y, Meyer EM, Walker DW, Millard WJ, He YJ, King MA (2002) Alpha7 nicotinic receptor activation inhibits ethanol-induced mitochondrial dysfunction, cytochrome c release and neurotoxicity in primary rat hippocampal neuronal cultures. J Neurochem 81: 853-858.

Logan GD, Cowan WB, Davis KA 1984 On the ability to inhibit responses in simple and choice reaction time tasks: a model and a method. J Exp Psychol: Human Percept Perform 10:276-291.

Madden PA, Heath AC (2002) Shared genetic vulnerability in alcohol and cigarette use and dependence. Alcohol Clin Exp Res 26:19191921.

Madden P, Heath A, Starmer G, Whitfield J, Martin N (1995) Alcohol sensitivity and smoking history in men and women. Alcohol Clin Exp Res 19:1111-1120.

Mason G, Petrakis I, deGraaf R, Appel M, Gueorguieva R, Guidone E, Coric V, Epperson CN E, Rothman D, Krystal J (2005) Brain GABA and recovery from alcohol dependence: preliminary finding of relationship with cigarette smoking. Biol Psychiatry [Epub ahead of print].

Mcmillan DE, Li M, Shide DJ (1999) Differences between alcohol-preferring and alcohol-nonpreferring rats in ethanol generalization. Pharmacol Biochem Behav 64:415-419.

Miller NS, Gold MS (1998) Comorbid cigarette and alcohol addiction: epidemiology and treatment. J Addict Dis 17:55-66 (Review).

Moriarty SE, Shah JH, Lynn M, Jiang S, Openo K, Jones DP, Sternberg P (2003) Oxidation of glutathione and cysteine in human plasma associated with smoking. Free Radic Biol Med 35:1582-1588.

Narahashi T, Aistrup GL, Marszalec W, Negata K (1999) Neuronal nicotinic acetylcholine receptors: a new target site of ethanol. Neurochem Int 35:131-141.

Narahashi T, Soderpalm B, Ericson M, Olausson P, Engel JA, Zhang X, Nordberg A, Marszalec W, Aistrup GL, Schmidt LG, Kalouti U, Smolka M, Hedlund L (2001) Mechanisms of alcohol-nicotine interactions: alcoholics versus smokers. Alcohol Clin Exp Res 25:152S$156 \mathrm{~S}$.

Nigg JT (1999) The ADHD response-inhibition deficit as measured by the stop task: replication with DSM-IV combined type, extension, and qualification. J Abnorm Child Psychol 27:393-402.

Nixon SJ, Paul R, Phillips M (1998) Cognitive efficiency in alcoholics and polysubstance abusers. Alcohol Clin Exp Res 22:1414-1420.

Nixon SJ, Tivis R, Parsons OA (1995) Behavioral dysfunction and cognitive efficiency in male and female alcoholics. Alcohol Clin Exp Res 19:577-581.

Peto R, Lopez AD, Boreham J, Thun M, Heath C Jr., Doll R (1996) Mortality from smoking worldwide. Br Med Bull 52:12-21.

Pierce RC, Kumaresan V (2005) The mesolimbic dopamine system: the final common pathway for the reinforcing effects of drug of abuse? Neurosci Behav Rev [Epub ahead of print].
Pomerleau CS, Aubin HJ, Pomerleau OF (1997) Self-reported alcohol use patterns in a sample of male and female heavy smokers. J Addict Dis 16:19-24.

Powell DH, Kaplan EF, Whitla D, Weintraub S, Catlin R, Funkenstein HH (1994) MicroCog: Assessment of Cognitive Functioning. Psychological Corporation, San Antonio, TX.

Prendergast MA, Harris BR, Mayer S, Littleton JM (2000) Chronic, but not acute, nicotine exposure attenuates ethanol withdrawal-induced hippocampal damage in vitro. Alcohol Clin Exp Res 24:1583-1592.

Rohsenow DJ, Monti PM, Colby SM, Gulliver SB, Swift RM, Abrams DB (2003) Naltrexone treatment for alcoholics: effect on cigarette smoking rates. Nicotine Tobacco Res 5:231-236.

Romberger DJ, Grant K (2004) Alcohol consumption and smoking status: the role of smoking cessation. Biomed Pharmacother 58:77-83.

Room R (2004) Smoking and drinking as complementary behaviours. Biomed Pharmacother 58:111-115.

Sattler JM (2001) Assessment of Children: Cognitive Applications. 4th ed. Sattler, J.M., San Diego.

Staley J, Gottschalk C, Petrakis I, Gueorguieva R, Baldwin R, Jatlow P, Verhoeff N, Perry E, Weinzimmer D, Frohlich E, Ruff E, Seibyl J, Innis R, Krystal J (2005) Cortical GABAA/benzodiazepine receptors in recovery from alcohol dependence: relationship to features of alcohol dependence and cigarette smoking. Arch Gen Psychiatry 62:877-888.

Sullivan EV, Pfefferbaum A (2005) Neurocircuitry in alcoholism: a substrate for disruption and repair. Psychopharmacology 180:583594.

Tarter RE, Edwards KL (1986) Multifactorial etiology of neuropsychological impairment in alcoholics. Alcohol Clin Exp Res 10:128-135.

Tizabi Y, Al-Namaeh M, Manaye KF, Taylor RE (2003) Protective effects of nicotine on ethanol-induced toxicity in cultured cerebellar granule cells. Neurotoxicity Res 5:315-322.

Tizabi Y, Copeland RL Jr., Louis VA, Taylor RE (2002) Effects of combined systemic alcohol and central nicotine administration into VTA on dopamine release in the nucleus accumbens. Alcohol Clin Exp Res 26:394-399.

Tizabi Y, Copeland RL Jr., Newton SS, Taylor RE (2004a) Effects of combined systemic alcohol and nicotine administration on dopamine release in the shell region of nucleus accumbens. Alcohol Clin Exp Res 28 (suppl): 40A

Tizabi Y, Getachew B, Davila-Garcia M, Taylor RE (2001) Alcohol preference: association with reduced striatal nicotinic receptors. Alcohol Alcoholism 36:318-322.

Tizabi Y, Manaye KF, Smoot DT, Taylor RE (2004b) Nicotine inhibits ethanol-induced toxicity in cultured cortical cells. Neurotoxicity Res 6:311-316

Wehner JM, Keller JJ, Keller AB, Picciotto MR, Paylor R, Booker TK, Beaudet A, Heinemann SF, Balogh SA (2004) Role of neuronal nicotinic receptors in the effects of nicotine and ethanol on contextual fear conditioning. Neuroscience 129:11-24.

Wu G, Miller KW (1994) Ethanol enhances agonist-induced fast desensitization in nicotinic acetylcholine receptors. Biochemistry 33:90859089

York JL, Hirsch JA (1995) Drinking patterns and health status in smoking and nonsmoking alcoholics. Alcohol Clin Exp Res 19:666-673.

Yoshida K, Engel J, Liljequist S (1982) The effect of chronic ethanol administration on high affinity $3 \mathrm{H}$-nicotine binding in rat brain. NaunSchmied Arch Pharmacol 321:74-76.

Young EM, Mahler S, Chi H, de Wit H (2005) Mecamylamine and ethanol preference in healthy volunteers. Alcohol Clin Exp Res 29: $58-65$.

Zucker RA, Ellis DE, Fitzgerald HE, Bingham CR, Sanford KP (1996) Other evidence for at lest two alcoholisms, II: life course variation in antisociality and heterogeneity of alcoholic outcome. Dev Psychopathol 8:831-848. 